\title{
SCÈNE ET CONTEMPORANÉITÉ
}

Frédéric Pouillaude

Collège international de Philosophie | « Rue Descartes »

$2004 / 2 n^{\circ} 44 \mid$ pages 8 à 20

ISSN 1144-0821

ISBN 9782130544562

Article disponible en ligne à l'adresse :

https://www.cairn.info/revue-rue-descartes-2004-2-page-8.htm

\section{Pour citer cet article :}

Frédéric Pouillaude, "Scène et contemporanéité », Rue Descartes 2004/2 ( $\left.\mathrm{n}^{\circ} 44\right)$, p. 8-20.

DOI 10.3917/rdes.044.0008

Distribution électronique Cairn.info pour Collège international de Philosophie.

(C) Collège international de Philosophie. Tous droits réservés pour tous pays.

La reproduction ou représentation de cet article, notamment par photocopie, n'est autorisée que dans les limites des conditions générales d'utilisation du site ou, le cas échéant, des conditions générales de la licence souscrite par votre établissement. Toute autre reproduction ou représentation, en tout ou partie, sous quelque forme et de quelque manière que ce soit, est interdite sauf accord préalable et écrit de l'éditeur, en dehors des cas prévus par la législation en vigueur en France. Il est précisé que son stockage dans une base de données est également interdit. 


\section{FRÉDÉRIC POUILLAUDE Scène et contemporanéité}

Du contemporain, il y aurait exposition. Et peut-être même scène.

Sur cette scène, éventuellement chorégraphique, c'est l'esprit du temps, la figure de l'époque, qui se donneraient obscurément à lire. Le temps humain aurait pour propriété essentielle de dessiner des images de lui-même, de s'auto-figurer, de se mettre en scène ; l'opération aurait pour médium privilégié certains produits traditionnellement nommés «œuvres d'art ».Si bien qu'ayant à parler de la danse et du contemporain, nous n'aurions plus qu'à tenter de déchiffrer dans les ouvres par elle produites et leur milieu d'existence-précisément, la scène-quelque chose comme des images du temps, de notre temps.

Telle est justement la voie que nous ne suivrons pas.

Pour deux raisons essentiellement. D'abord, - et nous faisons là qu'avancer une hypothèse qui déborde de très loin notre propos - ce que nous entendons aujourd'hui sous le terme vide de «contemporain» ne consiste peut-être en rien d'autre qu'en l'impossibilité avérée de la figuration époquale. Nous ne croyons plus à l'unité d'un temps qui soit nôtre, à la possibilité d'une présentation unifiante et figurée de notre temps. Les noms stratégiquement donnés à cette situation sont multiples et idéologiquement hétérogènes : «fin des grands récits », «fin des utopies», «mort de l'art», «post-modernité», «fin des avant-gardes», «fin de l'histoire »... Ce qui se laisse percevoir à travers la mélopée de la perte et l'hétérogénéité du discours élégiaque est peut-être plus déterminé qu'on ne le croit: ce n'est pas l'histoire comme telle et en général qui serait perdue, mais la capacité du temps, de «notre» temps, à se donner figure et à se saisir de lui-même comme d'un Un. En ce dispositif, la place de l'art, ou plutôt d'un certain diagnostic porté sur son état, est, comme on peut s'en douter, cruciale. Instance traditionnelle de figuration du temps et de présentation de l'époque, l'art doit logiquement se trouver au centre de la crise. Mort une première fois, avec Hegel, dans l'achèvement grandiose de sa mission onto-théologique, le spectre aurait survécu quelque temps à sa sécularisation. Se donnant d'autres tâches, il aurait trouvé dans le discours 
moderniste et la figure de l'avant-garde une forme de téléologie substitutive et athée, capable de garantir son inscription historico-politique et sa responsabilité devant le temps. Mais la substitution aurait elle-même fait long feu ; le spectre serait aujourd'hui bel et bien mort, non par achèvement de sa mission, mais par évanouissement de toute structure téléologique capable de lui assigner une fonction. L'art serait entré en régime «gazeux» ${ }^{\mathbf{1}}$, délié de toute responsabilité, flottant parmi les infinis possibles, et ne répondant à d'autre nécessité qu'au jeu de la reconnaissance institutionnelle, de la nouveauté et de la subversion vides, immédiatement intégrées et recyclées par le marché ou l'État. L'art aurait perdu toute puissance de figuration époquale, et se contenterait, au même titre que n'importe quel secteur économique, de jouer le jeu social qui est le sien. Ni plus, ni moins. Si bien que « contemporain » signifierait à la fois l'incapacité générale de notre temps à se saisir et figurer comme époque, et le cœur local d'une telle incapacité, sa cause peut-être : une certaine crise de la production artistique ou, du moins, son entrée dans un régime tellement autre qu'on ne pourrait pour l'instant le penser qu'à partir de ce qui s'en est absenté. De sorte qu'il serait bien absurde de vouloir encore appliquer à l'art, et en l'occurrence à la danse, ce schème de la figuration époquale que le terme même de «contemporain » semble doublement renier. Telle serait la première raison de notre refus, à vrai dire extérieure et insuffisante.

En effet, il n'est pas si facile d'échapper à la logique de l'époqualité. C'est encore sous forme d'époque, même vide, qu' est présentée l'incapacité de notre temps à se saisir de lui-même, et c'est encore sous forme de figure temporelle, homogène et unifiée, quoique sans doute négative, qu'est appréhendée notre impossibilité à constituer «un» temps «nôtre». Le propre de «notre temps» serait de n'en être ni un, ni le nôtre. Mais ce «propre», justement en tant que propre, permettrait malgré tout de continuer à en parler comme d'un Un et de nous l'attribuer, à nous, quand bien même ce «nous » ne désignerait rien d'autre que sa propre impossibilité. «Contemporain», dans sa forme nominale et unifiante, serait précisément l'emblème d'une telle contradiction. «Contemporain » serait un nom restant, qui simultanément rêve et annule l'unification dans le Zeitgeist, qui annule le Zeitgeist tout en conservant la place vide de son sujet. Nous ne croyons certes plus à l'idée d'un temps unifié et commun, à l'idée d'une communauté substantielle du temps, mais la forme de notre incrédulité est encore une façon d'y souscrire. Par quoi, soit dit en passant, nous sommes encore pieux. Mais alors la figuration doit elle aussi, de quelque façon, continuer d'opérer. Et, de fait, on ne peut nier l'adéquation parfaite qui relie le nouveau régime de l'art à l'impossibilité 


\section{0 | FRÉDÉRIC POUILLAUDE}

contemporaine de l'autofiguration. «Contemporain», en ce qu'il désigne simultanément l'époque et ses productions artistiques, ne postule en fait rien d'autre qu'une telle adéquation comprise comme forme restante et négative de la figuration positive: dans l'incapacité contemporaine de l'art à donner forme au temps, c'est encore l'époque, l'esprit du temps, qui se donnerait négativement à lire. De sorte que notre refus serait encore trop naif. Malgré l'obsolescence d'une lecture positive des figures du temps dans l'art, une voie négative resterait disponible, sous deux formes. Soit l'on cherche à montrer, en intériorité, que l'art contemporain («post-moderne», si l'on veut) s'est donné pour tâche cssentielle de figurer l'impossibilité de la figuration, de présenter le fait qu'il y a de l'imprésentable, et l'on soutient que c'est en cela même qu'il parvient à représenter l'époque. Comme Lyotard le reconnaît lui-même, cette figuration négative reste solidaire de la logique moderniste et interne à l'opération artistique ${ }^{2}$. Soit, à l'inverse, on se contente de mettre au jour, en extériorité, un renoncement pur et simple de l'art, un abandon ou un évanouissement de sa tâche historique, et l'on fait de ce renoncement même la figure symptomatique, et très accessoirement artistique, de notre temps : il y aurait encore dans l'art figuration du temps, mais uniquement selon l'extériorité du symptôme, et non l'intériorité négative du symbole. Telle serait la voie culturelle et sociologique, où le thème général de la «mort de l'art», de la «fin de son mythe », etc., s'articule nécessairement à l'exhibition brute de son fonctionnement factuel et socio-économique. Les travaux d'Arthur Danto, de Nathalie Heinich, ou d'Yves Michaud, pourraient de façon très diverse constituer les jalons de cette seconde voie, «en extériorité ». ${ }^{3}$ Nous pourrions essayer d'appliquer ce schème de la figuration négative au champ chorégraphique. Nous pourrions montrer que la danse est, elle aussi, notamment par son usage non iconique du corps, expérience de l'imprésentable, présentation de l'imprésentabilité. Nous pourrions à l'inverse chercher à dégonfler des baudruches, montrer que le «champ» chorégraphique est lui aussi le lieu de la déliaison et de l'irresponsabilité artistiques, de l'éclectisme et de la singularité insignifiantes, et qu'en somme le jeu social de la subversion et de la reconnaissance vides s'y joue aussi bien qu'ailleurs. Nous ne le ferons pas. Et ce pour la seconde raison annoncée : c'est qu'en procédant ainsi nous n'apprendrions à vrai dire rien que nous ne sachions déjà, et en tout cas, rien de bien spécifique à la danse. Une

2. Voir Jean-François Lyotard, Le postmoderne expliqué aux enfants. Paris. Galilee. 1988. «Une æuvre ne peut devenir moderne que si elle est d'abord postmoderne. Le postmodernisme ainsi entendu n'est pas le moderni sme à sa fin, mais à 1 'état naissant, et cet état est constant. [...] Le postmoderne serait ce qui dans le moderne allègue 1 'imprésentable dans la présentation elle-même.» |3. Outre l'ouvrage d'Yves Michaud déjà cité. 11 faut mentionner (du même) La crise de 7 'art contemporain. Paris. PUF. 1997, ainsi que Natha 1 ie Heinich, Le triple jeu de l'art contempora in, Paris, Minuit, 1998, et Arthur Danto. Après la fin de l'art, trad. fr., Paris, Seuil, 1996. 
telle approche resterait extérieure à son objet, se contentant de lui appliquer un réseau de concepts et de problématiques issu d'un tout autre champ, généralement celui des arts plastiques et de la littérature. Sans doute saisirait-elle quelque chose de la danse, mais uniquement ce qui en elle participe très généralement de ce régime de l'art aujourd'hui nôtre. De sorte que la spécificité même de cette participation ne pourrait être prise en vue. C'est pourquoi nous proposons de repartir d'ailleurs, et de faire momentanément comme si nous ne savions rien de tout cela.

Mettant de côté le schème de la figuration époquale, nous partirons d'un certain nouage interne de la danse à la contemporanéité. «Contemporanéité» ne désigne pas ici une figure historique, une époque, mais une structure de temporalité. En l'occurrence : une simultanéité neutre, une coexistence contingence. Est «contemporain», en son sens le plus général et sans qu'aucune époque soit par là désignée, tout ce qui coexiste, tout ce qui appartient à un même temps. Nul besoin de fonder cette coexistence sur un partage de traits ou d'identités, sur une quelconque forme de communauté substantielle. Les choses, les personnes, les événements coexistent. Un point, c'est tout. Il n'y a pas à en rendre raison plus avant.

Comme on l'a dit, la simultanéité est neutre et la coexistence contingente. Mais, en même temps, précisément parce qu'elle autorise le rassemblement des coexistences sous un vocable unique (l'«époque», le «temps», le «contemporain»), la contemporanéité semble appeler au dépassement de la contingence : il faut bien qu'à un moment ou un autre la simultanéité rende compte d'elle-même et s'instaure en communauté véritable, il faut bien qu'une identité, une raison, un contenu, explique le fait de la coexistence, et le réassure comme relevant d'un présent réellement commun. De sorte que la contemporanéité serait à entendre de la façon suivante : une simultanéité neutre se marquant elle-même d'insuffisance et aspirant à l'unité d'une communauté substantielle. Nous soutiendrons que la danse, en tant qu'événement scénique, est directement travaillée par une telle structure. Ses œuvres propres n'ont d'autre milieu d'existence que la scène, et celle-ci, comme nous espérons le montrer, n'est pas autre chose qu'une structure de contemporanéité. De là, nous reviendrons au « contemporain » lui-même, et exposerons une certaine mutation intervenue dans le champ chorégraphique au cours des dix dernières années. Nous soutiendrons que cette mutation ne peut véritablement s'entendre qu'en référence à la contemporanéité de la scène, dont elle serait, en tant que travail réflexif de la performance, prise de conscience et remise en jeu radicale. 


\section{2 | FRÉDÉRIC POUILLAUDE}

Commençons par un axiome simple, quoiqu'en apparence exorbitant : il n'y a à proprement parler de danse que contemporaine. Non pas, évidemment, qu'il faille considérer le genre «danse contemporaine» comme terminus ad quem de l'histoire chorégraphique, comme moment d'assomption où l'expérience du mouvement se trouverait relevée en son ultime figure de vérité, mais, plus simplement, soutenir une évidence minimale, à savoir : que la danse ne s'est jamais donnée autrement que de présence à présence, dans un espace de simultanéité à soi et aux autres qui est précisément ce que l'on nomme une scène et qui a tous les aspects d'une structure de contemporanéité. On objectera sans doute que la scène n'est nullement nécessaire à l'expérience de la danse. On peut fort bien danser pour soi-même, sans s'adresser à qui que ce soit. Et, à supposer que quelqu'un d'autre soit présent, il faut bien reconnaître que celui-ci est le plus souvent également dansant, tout participatif et pas du tout assis. Soit. Il n'en demeure pas moins que c'est uniquement à travers la structure scénique que la danse peut se constituer et s'offrir en ouvre. Tant qu'elle reste auprès d'elle-même, repliée dans l'expérience intime de son geste, rien ne s'en dégage qui puisse assumer la figure de l'objet (si ce n'est par l'entremise d'un regard indélicat et voyeur instaurant en spectacle ce qui n'est nullement fait pour être vu). C'est seulement par l'adresse intentionnelle et le partage explicite des actants et des regardants qu'un tiers objet peut s'extraire, ouvrant la possibilité d'une auvre. Et s'il s'agit de parler ici de la danse comme d'un art, comme d'un mode de production et de présentation d'objets par où ceux qui font et ceux qui ne font pas en viennent à se rencontrer, il nous faut alors sans scrupules reconduire cette réduction de la danse à la scène.

Par cette réduction, c'est encore le nouage de la danse à la contemporanéité qui est affirmé. Car l'expérience solipsiste du mouvement n'est nullement concernée par la contemporanéité. Son temps est certes celui de la présence, mais d'une présence toute repliée sur elle-même, sans articulation dialectique aucune, sphère parfaite d'auto-affections pures. Déployant en soi et pour soi la suite de ses propres kinesthésies, le corps dansant n'a pour temporalité qu'un présent d'éternité d'où s'est absentée toute coexistence possible. Danser à plusieurs ne changera sans doute rien à l'affaire. On ne fera que décaler d'un cran le sujet global : on sera simplement solipsiste en groupe, en une forme fusionnelle et non-dialectique de l'expérience du commun ${ }^{4}$. Pour que la contemporanéité puisse véritablement s'expérimenter, la coexistence ne doit pas se résorber immédiatement en fusion sans dehors ; il faut qu'un hiatus se réintroduise, et qu'apparaisse le clivage de la scène.

Réduisons donc la danse à la scène et faisons de cette dernière l'emblème d'une articulation possible entre danse et contemporanéité. Rappelons cependant que nous ne savons toujours

4. Il faudrait ici immensément nuancer. la création contemporaine étant en grande partie orientée par la recherche d'une forme non fusionnelle de communauté. Sur ce point, voir Les lieux de là de Mathilde Monnier (1999), qui figure comme le coup d'envoi d'une telle recherche. 
pas ce qu'est une scène et comment cela fonctionne. Essayons alors de décrire la chose, en une tentative très faussement phénoménologique.

Des gens sont là, réunis en multiplicité indifférente, sans autre raison que d'y être. Une sorte de tas local. Ils ne font rien, ou pas grand chose, ou bien pas vraiment. Ils attendent quelque événement. D'autres, en face, ou derrière, ou à côté, ou bien même parmi eux-c'est indifférent -, font que quelque chose advienne, que quelque chose s'effectue, éventuellement sous forme de rien, mais en tout cas d'une façon suffisamment claire pour se dégager du tas et qu'en retour les premiers, le tas justement, se constituent en public. Peut-être allons-nous trop vite en avançant ainsi le terme de «public». Peut-être ne fait-il que masquer le phénomène qui le sous-tend. Tout ce que nous savons pour l'instant, c'est que d'un côté des gens attendent et ne font rien, et que de l'autre certains agissent, effectuent, s'agitent (ou pas). Surtout, nous savons que ces deux choses doivent arriver en même temps, selon une forme de coexistence qu'il nous faut bien pour l'instant qualifier de contingente. Ceux qui font et ceux qui ne font pas n'ont à vrai dire aucune raison d'y être, et encore moins d'y être ensemble Un certain événement, celui-là même au nom de quoi se délimite la scène, ouvre un espace de coexistence, instaure un «nous» de simultanéité. Mais ce «nous» n'a pour l'instant d'autre contenu que celui de l'attente et de son remplissement mécanique. De sorte qu'en réalité rien n'est fait. L'attente ne peut se satisfaire de l'événement nu, de ce qui, arrivant ici et maintenant, a pour seul mérite son ecceité. Il faut qu'un supplément advienne. La présence seule ne suffit pas. Car nous y baignons déjà intégralement : ce qui se donne à voir, là-bas, sur la scène, le fait comme authentique première main, induisant chez tous la conscience très lucide que rien ne s'en répètera jamais à l'identique, où que ce soit, et, de ce fait, s'enrubanne d'aura. Mais l'on sait bien qu'une telle présence est factice, qu'une telle aura est mécaniquement induite par le désoeuvrement du tas et la vacuité son temps. Il faut en passer par là, c'est condition indépassable, et l'on y trouve même un certain plaisir. Pourtant c'est encore autre chose que l'on attend. On attend que la coexistence de ceux qui font, de ceux qui ne font pas, et de leur ensemble, échappe enfin à la pure absence de fondement en laquelle elle s'origine, et se réinstaure autour d'un «commun » quelconque. On attend que de la Vérité, de la Présence (un supplément de présence, ou plutôt une transsubstantiation de « $\mathrm{p}$ » à «P») rachètent un tant soit peu la contingence initiale et la redonnent sous forme de communauté avérée, de présent réellement partagé. Les applaudissements, qui à l'arrivée boucleront l'affaire, sont comme l'indice retardé d'une telle espérance, son ultime et décevant substitut. La scène n'est 


\section{I FRÉDÉRIC POUILLAUDE}

rien d'autre qu'une structure de contemporanéité, intensifiée et complexifiée. La coexistence s'y donne pliée en deux, compliquée du désoeuvrement des uns et de l'adresse intentionnelle des autres ; la contingence y est thématisée d'emblée, pour soi et non plus simplement en soi.

Soit. Mais en quoi cela concerne-t-il directement la danse? La structure scénique ne vaut-elle pas tout autant pour le théâtre ou le concert? Certes. À cette différence près que l'œurre chorégraphique, au contraire de l'œuvre musicale ou théâtrale, n'a d'autre lieu de subsistance que la scène même, et qu'y accéder, c'est nécessairement participer à sa donation en présence. Et ce pour deux raisons. D'abord, l'œuvre chorégraphique n'érige pas en condition d'identité un élément particulier de la performance. Alors que l'identité d'un opéra ou d'une pièce de théâtre n'est définie que par un seul de ses champs et peut de ce fait donner lieu à diverses interprétations et mises en scène, le spectacle de danse conserve l'hétérogénéité des éléments spectaculaires sans la hiérarchiser. Tout participe alors à la définition de l'œuvre, y compris ces éléments apparemment secondaires que sont les décors, les costumes ou les éclairages. Ainsi, parler des Trois boléros d'Odile Duboc, c'est non seulement renvoyer à la chorégraphie de Duboc et à la musique de Ravel, mais aussi bien aux costumes de Dominique Fabrègue, aux décors de Sélim Saïah, aux éclairages de Françoise Michel. De sorte qu'une telle œuvre reste radicalement dérobée hors la rencontre événementielle et scénique des champs qui la constituent. Les Ballets russes sont comme le point d'origine et le paradigme de cette événementialité de l'œuvre hétérogène, fort bien décrite par Jankélévitch : «Il n'y pas d'“œuvres” des Ballets Russes, mais il y a au point de jonction de plusieurs arts, une œuvre pneumatique qui est paradoxalement une œuvre de l'instant, qui apparaît et disparaît au cours de la même soirée, sans laisser d'elle-même aucun dépôt dans les archives en dehors d'un enregistrement électromagnétique plus ou moins illusoire. Cette "ouvre" instantanée est la conjonction miraculeuse d'une musique, d'une chorégraphie et d'un décor ; et on peut la dire "semelfactive", en ce sens que, même répétée, elle apparait chaque fois pour la première fois, chaque fois pour la dernière, chaque fois pour la première-dernière fois $\gg^{\mathbf{5}}{ }^{5}$. Ne résorbant pas l'hétérogénéité du spectacle en un élément unique, l'œuvre chorégraphique ne peut subsister qu'à même la scène, dans la temporalité «semelfactive » de l'événement et la contemporanéité des actants et des regardants.

D'autre part, à supposer que l'œuvre puisse se définir depuis la seule identité du texte chorégraphique, il faut encore noter le caractère ambigu et mal assuré de ce texte même, très généralement oral. Peu de danseurs ou de chorégraphes savent lire ou écrire une partition chorégraphique.

5. Jankékévitch, V., Liszt et la rhapsodie, t. 1. Paris. Plon, 1979, « Le mystère de la temporali té ». p. 167. 
La notation, lorsqu'elle a lieu (ce qui est rare), fonctionne plus comme un dispositif de consignation extérieure (une fixation d'archive) que comme un principe d'identification autorisant des reprises étrangères aux transmissions personnelles (un corpus). La notation ne s'étant jamais réellement intégrée aux pratiques, le passage à l'allographie qui s'annonçait en elle (par quoi l'œuvre aurait pu s'abstraire de ses conditions locales de production et de transmission) est resté lettre morte. Les ouvres ont continué à se transmettre de façon massivement orale, de corps à corps, de présence à présence. La composition même est restée soumise à cet impératif : inconcevable dans l'espace abstrait et solitaire de la page, elle ne peut advenir qu'à même le corps singulier d'interprètes choisis, oeuvrant d'emblée dans l'espace de co-présence du studio. Ne disposant pas des procédures d'absence autorisées par le texte, la danse s'indexe à un horizon de présence généralisée. De sorte que là encore sa donation reste vouée à la contemporanéité.

Les années quatre-vingts furent en France comme l'oubli d'une telle dépendance. Plus exactement, sa mise entre parenthèses, non questionnée. Car c'est d'abord comme réitérabilité indifférente que se définissait une œuvre. Les pièces, s'inscrivant au répertoire des compagnies, se voulaient indépendantes de leurs conditions locales d'actualisation et supportaient sans broncher le calendrier hétérogène des soirées et des tournées. Des œuvres tout-terrain, en quelque sorte, jouant à nier l'événementialité de leur donation et à s'en abstraire. Un soir ici, un soir là-bas, dans tel théâtre, dans tel autre, et pourtant, imperturbablement, la même œuvre. Cette mise entre parenthèses du fait de la performance eut pour conséquence l'absence de remise en question de sa forme même. La structure scénique et ses paramètres furent traités selon l'évidence d'un médium indépassable et non questionné. Ce que l'on nommait encore à l'époque « spectacle de danse » semblait aller de soi, et l'essence de la performance - faire quelque chose en temps réel devant des gens qui ne font rien - ne pas poser plus de difficultés que cela.

La mutation dont nous parlons (que l'on pourrait dater du milieu des années quatre-vingt-dix) doit s'entendre comme l'exact contre-pied de cet état. Diversement nommée par la critique, qui la réduit à l'état d'avant-garde locale («Nouvelle scène française », « Jeune danse », etc.) ${ }^{6}$, elle constitue, quel que ce soit le jugement qu'on porte sur elle, plus qu'une mode ou une tendance passagère ; ce qui s'annonce en elle, c'est concernant la production des ouvres un changement radical de régime. Et, à vrai dire, ce changement est déjà advenu. Il y aujourd'hui, pour quiconque entend œuvrer en danse, de l'impossible, des choses qu'on ne peut tout

6. Sans chercher à dresser un catalogue, donnons néanmoins quelques noms : Alain Buffard, Jérôme Bel. Boris Charmatz, Emmanuelle Huynh, Xavier Leroy, Ala in Michard, Laurend Pichaud, Loīc Touzé... 


\section{6 | FRÉDÉRIC POUILLAUDE}

simplement plus faire, ou du moins plus avec la même naïveté.Quels sont les traits de cette mutation? Pour la clarté de l'analyse, nous en isolerons cinq, lesquels peuvent se subsumer en un syntagme unique : «travail réflexif de la performance».

Le premier trait consiste dans la dissolution des compagnies fixes. À l'équipe des collaborateurs stables et salariés-ce qu'on appelait jadis une «compagnic»-, se sont substituées des coalitions temporaires et locales, réunissant autour d'un projet défini des individus menant de façon autonome leur propre carrière artistique. Ce modèle, à la fois libéral et libertaire, référant le travail au caractère temporaire de la mission et au consentement circonscrit de ses participants, ne fait pas que répondre à des motivations d'ordre économique. La dissolution de la compagnie Mathilde Monnier, en 1999, est là pour le prouver. Effectuée hors d'une réelle nécessité financière (la compagnie bénéficiait de la renommée, et donc des subventions, dues à tout Centre chorégraphique national), la dissolution tentait de répondre à la situation d'impasse créée par le travail salarié au sein de la compagnie (à ce que Mathilde Monnier nomme la «névrose familiale»), et à la conscience lucide que l'engagement réciproque du danseur et du chorégraphe ne pouvait excéder l'adéquation locale à tel ou tel projet. La précarité du travail devient alors norme artistique interne. Le contrat à durée indéterminée (ou du moins l'engagement sur un temps long) n'est plus ce dont il faut déplorer l'absence, mais au contraire ce qui a été rejeté pour des raisons internes à la production artistique. De sorte que le régime de l'intermittence ne vient pas pallier un salariat idéal que chacun devrait s'efforcer de rejoindre, mais ne fait qu'accompagner socialement, et de façon absolument indispensable, un libéralisme intégralement assumé par les acteurs. Il faudrait ici citer la formule exemplaire de Boris Charmatz, où l'intermittence se définit comme "précarité (sociale) assumée au bénéfice des échanges (artistiques)» ${ }^{7}$, le sel de la formule résidant bien entendu dans le jeu des parenthèses : une fois éliminée la parenthèse «artistique», que reste-t-il sinon l'une des formulations possibles du programme économique du Medef ${ }^{8}$ Cette mutation du travail n'est pas sans conséquence sur le conccpt même d'œuvre. Deux motifs liés s'y affaiblissent : l'auteur et le répertoire. L'œuvre cesse d'être le produit intégral du chorégraphe pour

7. Boris Charmatz et Isabelle Launay, Entretenir, Paris. Centre national de la danse, 2003, p. 139. Intermittence, mon amour, Édition spéciale de la revue Mouvement. juillet 2003. téléchargeable à 1 'adresse suivante : http://www.mouvement. net/Intermittence.pdf - Nous avons lu le protocole, film réal isé par un collectif $d^{\prime}$ artistes et de techniciens intermittents du spectacle, qui analyse de façon remarquable les différents mécanismes d'exclusion mis en place par la réforme, téléchargeable à 1 adresse suivante : http://video.protocole.free.fr - Site de la coordinatioon des intermittents et précaires d'Ile-de-France : http://cip-idf.ouvaton.org - Site des précaires associés de Paris : http://pap.ouvaton.org 
devenir le résultat local et temporaire d'une coalition qui ne l'est pas moins : «Des artistes ont consenti à travailler ensemble pour un temps donné, et voici le résultat... » ${ }^{9}$. Par là, c'est aussi la notion de répertoire qui disparaît, puisque l'ouvre ne peut que difficilement subsister au-delà de la coalition qui l'autorise. Les œuvres ne se constituent en répertoire que si la signature de l'auteur suffit seule à les identifier et qu'une compagnie fixe, précisément liée au nom du chorégraphe, est là pour les actualiser. Cette mutation générale du travail chorégraphique (qui est aussi mutation de l'œuvre), nous l'interprétons comme la prise de conscience du caractère nécessairement local et temporaire de la performance, comme la prise en vue de son indépassable contemporanéité, laquelle restait oblitérée par la structure de la «compagnie » et du « répertoire».

Le second trait consiste dans l'intégration des œuvres à leur contexte économique de production et de diffusion. Il s'agit de traiter les paramètres financiers de la création, non comme des éléments secondaires et contingents sur lesquels il faudrait jeter un voile pudique, mais au contraire comme des traits propres et constitutifs. On entend adapter l'œuvre, à chaque fois de façon différente, aux lieux et aux structures qui l'accueillent et la financent. Par là, on abandonne la figure universelle de l'cuvre tout-terrain qui, s'abstrayant de ses conditions matérielles de production, présidait aux années quatre-vingts. La mutation consiste, économiquement parlant, à fondre ensemble production et diffusion. Dans la production, les partenaires donnent de l'argent pour qu'une nouvelle ceuvre soit créée. Dans la diffusion, ils se contentent d'acheter une pièce déjà faite qu'il s'agira simplement d'actualiser sur le plateau. On exige aujourd'hui que chaque temps de diffusion (d'actualisation de l'œuvre) soit simultanément un temps de production (de recréation). Bords d'Emmanuelle Huynh, est l'exemple parfait de cette fusion. Cette pièce se métamorphose en chacune de ses actualisations et ne se vend qu'à la condition expresse qu'un temps de création soit dégagé pour chaque diffusion. Là encore, il ne s'agit pour la performance que d'être à la hauteur de sa nécessaire contemporanéité.

Le troisième trait repose dans la mutation du concept d' «écriture » (lequel ne renvoie pas à la notation graphique, mais à la composition: il désigne alors l'ensemble des procédures

8. Sur la mutation 1 ibérale des arts du spectacle, pensée dans son rapport à la précarisation générale du travail au sein de l'économie «ordinaire», nous renvoyons aux remarquables analyses de PierreMichel Menger dans Portrait de l'artiste en travailleur. Metamorphoses du capitalisme. Paris. Seuil, 2002 , et notamment au chapitre 3 «Les arts : laboratoire de la flexibilité». |9. Le projet d'Alain Buffard pour Montpellier-Danse 2003, Mauvais genre, annulé pour cause de grève des artistes, est exemplaire d'un tel dispositif. Il devait réunir une trentaine de chorégraphes-danseurs, disposant déjà d'une solide notoriété et menant par ailleurs leurs propres travaux : Régine Chopinot. Xavier Leroy. Mathilde Monnier, Rachid Ouramdane, Mark Tompkins, etc. 


\section{FRÉDÉRIC POUILLAUDE}

permettant d'identifier et de fixer l'objet chorégraphique en une entité stable et réitérable). L'écriture chorégraphique ne consiste plus aujourd'hui dans la fixation d'un tracé gestuel déterminé que l'interprète aurait à actualiser mécaniquement d'un soir à l'autre, mais dans la mise en place de dispositifs ouverts exigeant d'être rééxpérimentés et retraversés de façon différente à chaque occurrence. L'ouverture de l'écriture implique alors qu'une part d'improvisation se loge en chaque temps d'actualisation, de sorte que l'événement scénique cesse précisément d'être actualisation mécanique et devient recréation partielle. L'écriture par matière, se contentant de déterminer les paramètres généraux de l'identité du geste sans fixer sa forme, constitue l'une des modalités possibles de cette écriture renouvelée. Quoique fort généralisée aujourd'hui, on trouverait un cas exemplaire dans le travail de Mathilde Monnier, et notamment Multi-materials et Déroutes. Par cctte mutation de l'écriture, l'œuvre ne fait là encore que s'aligner sur la nécessaire événementialité de sa donation.

Le quatrième trait consiste dans la perte d'évidence du médium «danse». Les années quatrevingts ont vécu dans un relatif consensus concernant l'identité de la danse. Malgré la diversité des styles, une démarcation évidente à l'égard du mouvement « piéton » et quotidien restait comme constitutive et inattaquée. Cettc évidence est morte. Tout peut aujourd'hui être de la danse, y compris (et sans doute surtout) le geste le plus banal ou même le plus absent et immobile. Cette remise en question de l'identité du chorégraphique peut se lire comme un

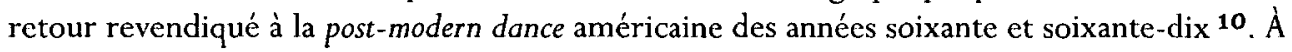
une différence près. Tandis que la post-modern dance se comprenait comme le questionnement analytique d'une essence (Qu'est-ce que la danse ?), opérant par variation éidétique et passage à la limite (Jusqu'où peut-on aller sans cesser de produire de la danse ?), notre mutation semble plutôt décaler le questionnement vers l'essence de la performance (Jusqu'où y a-t-il événement lorsque je fais quelque chose-ou pas - devant quelqu'un qui ne fait rien?). C'est seulement depuis cette mise en question de la performance que le médium « danse » est à son tour réinterrogé. Muà d'Emmanuelle Huynh est là pour le prouver. Dans ce solo fait tout entier d'immobilité, de silence et d'obscurité, ce n'est qu'à travers une remise en cause générale des paramètres spectaculaires que la danse en vient à se questionner.

De sorte que notre cinquième trait-l'opacification réflexive du médium «spectacle »-constitue en réalité la vérité du précédent. Les années quatre-vingts ont œuvré dans une franche naïveté à l'égard de la forme spectaculaire, la traitant comme médium indépassable, neutre et transparent. À travers ce médium, des «mondes» chorégraphiques

10. Sur ce lien entre la «Nouvelle scène » et la post-modern dance américaine, nous renvoyons aux analyses de Denise Luccioni, dans son avant-propos à 1 a traduction française de 1 'ouvrage de Sally banes Terpsichore en basket. Post-modern dance. Paris. Centre national de la danse, 2002, p. $10 \ll$ Un miroir tendu à la danse française actuelle». 
s'exhibaient directement, lointains et autonomes, inaltérés par la relation et indifférents au fait de se donner. Le spectacle n'était jamais qu'un coin de visibilité offert sur eux, et ne les modifiait nullement. Au sein de ce dispositif, des œuvres mémorables furent produites. Tel May Be de Maguy Marin, univers beckettien clos sur lui-même, s'offrant simplement aux regards, sans que jamais la donation se thématise. La chose est devenue impossible. Il y aurait aujourd'hui en forme d'axiome contemporain comme une nécessaire auto-réflexivité de la performance. Le spectacle ne pourrait échapper à son essentielle duplicité et putasserie qu'en thématisant sur la scène son opération et en devenant à soi son propre objet. Hors la réflexivité du médium spectaculaire, point de salut! Les pièces de Jérome Bel, dont les titres (Le dernier spectacle, The show must go on,...) parlent d'eux-mêmes, sont l'exemple éclatant d'un tel impératif.

Ces cinq traits peuvent se subsumer en un syntagme unique: «travail réflexif de la performance». L'événementialité de la performance en vient à se prendre en vue comme telle, au point de devenir immanente au concept d'œuvre (points 1, 2, 3). La donation spectaculaire devient à elle-même son propre objet et s'autorise par là des formes de remise en cause interne (points 4 et 5). Notre geste consiste alors à pousser plus loin l'argument, en référant une telle mutation à la contemporanéité dialectique de la scène, dont elle serait à la fois prise de conscience et remise en jeu radicale.

On est en droit de s'interroger sur le statut historique d'une telle mutation. Nous avions cru en préalable pouvoir mettre de côté toute question d'époqualité. En un juste retour des choses, nous serions maintenant comme rattrapés. Cependant, là encore, nous ne répondrons que négativement. Nous soutiendrons que cette mutation échappe aux termes ordinairement en usage pour penser l'historicité des arts. Elle n'est ni moderne, ni postmoderne. Elle ne consiste pas, comme le veut le modernisme, dans un acheminement de l'art vers son propre : ce n'est pas la «danse» comme telle qui fait l'objet de la réflexion, mais plutôt la performance, laquelle n'est qu'accidentellement reliée à la danse et ne figure nullement un propre (il aurait suffit que la notation passe réellement dans les mœurs pour que la dépendance de la danse à la performance soit considérablement affaiblie). De plus, cette mutation ne fait que répéter et décaler une mutation déjà advenue (celle de la «postmodern dance» américaine), de sorte que la répétition vient briser les figures de progrès et de dépassement successif des formes propres à la logique moderniste. Mais elle n'est pas pour autant postmoderne. La répétition y possède une réelle consistance interne, qui l'empêche de verser dans le simple éclectisme du « retour à » et la fait échapper au pur évanouissement de 
l'historicité que suppose la postmodernité. Par ailleurs, la «postmodern dance» américaine qu'elle répète est en réalité tout sauf « postmoderne ». Car les noms historiques dont la danse s'affuble sont comme décalés quant à leur contenu artistique. En effet, au risque du paradoxe, il nous faut soutenir que la danse « moderne » (Laban, Wigman, Graham...) est classique : elle est tout entière ordonnée selon l'expressivité d'un sujet créateur. Et, parallèlement, que la "postmodern dance» américaine est moderne: elle s'achemine, par passage à la limite et outrepassement de ce qu'elle est censée être, vers l'élucidation de son propre ${ }^{11}$. Notre mutation, quant à elle, ne serait ni moderne, ni postmoderne. Elle serait contemporaine, en un sens extra- ou para-historique. Elle tenterait de prendre en vue la nécessaire contemporanéité de la performance (laquelle n'est pas un propre mais un accident); elle essaierait de réfléchir dans son œuvre même ce que c'est que de s'exposer à la coexistence contingente de ceux qui font et de ceux qui ne font pas. Deux motifs sont alors noués : la présence (la nécessaire événementialité de ce qui ne peut se donner qu'en acte), et la contingence (l'insuffisance dialectique de la simultanéité neutre), motifs que notre mutation tente d'élever à la conscience de soi. L'œuvre ne s'abstrait plus de l'événementialité de sa donation, et la contingence en vient à se thématiser pour soi, à travers des formes de résorption essentiellement déceptives ou absentes (il s'agira de produire des événements nuls, des danses invisibles, des gestes immobiles, et d'aller ainsi contre la satisfaction de toute attente). Par là, c'est comme une définition immanente de la performance qui s'offre à nous : une chose qui n'est possible qu'en présence, et sous condition de son ratage.

Frédéric Pouillaude enseigne la philosophie à l'université Lille Ill et prépare une thèse sur la danse contemporaine. II est l'auteur de plusieurs articles, dont «D'une graphie qui ne dit rien : les ambiguïtés de la notation chorégraphique» à paraître en 2004 dans la revue Poétique. Il est ancien élève de Ex.e.r.c.e. (CCN de Montpellier)

11 Nous nous appuyons ici sur les analyses de Sally Banes, Terpsichore en Baskets. tr. fr.. op. cit.. p. 19 : «La modern dance historique $n$ ' a jama is vraiment été moderniste. Souvent, les questions soulevées par le modernisme dans les autres arts surgissent précisément dans 1 'arène de la post-modern dance: 1 'inventaire des propriétés du médium. $l$ exposition des qualités essentielles de la danse considérée comme un art. la dissociation des éléments formels. l'élimination de tout contenu extérieur à la danse. Aussi. sur bien des points. c'est la post-modern dance qui a une démarche moderniste. [..] Puisque «moderne»appliqué à la danse $n$ 'est pas synonyme de «moderniste». être anti-modern dance ne signifie pas du tout être anti-moderniste. bien au contrai re.» 